\title{
The Quick Sequential Organ Failure Assessment (qSOFA) Score is a Poor Mortality Predictor in Patients with Complicated Intra-abdominal Infections
}

\author{
Evgeni Dimitrov $^{1 *}$, Georgi Minkov${ }^{1}$, Emil Enchev $^{1}$, Krasimira Halacheva $^{2}$, Yovcho Yovtchev $^{1}$ \\ ${ }^{1}$ Department of Surgical Diseases, University Hospital "Prof. Dr. Stoyan Kirkovich," Stara Zagora, Bulgaria; ${ }^{2}$ Department of \\ Immunology, Faculty of Medicine, Trakia University, Stara Zagora, Bulgaria
}

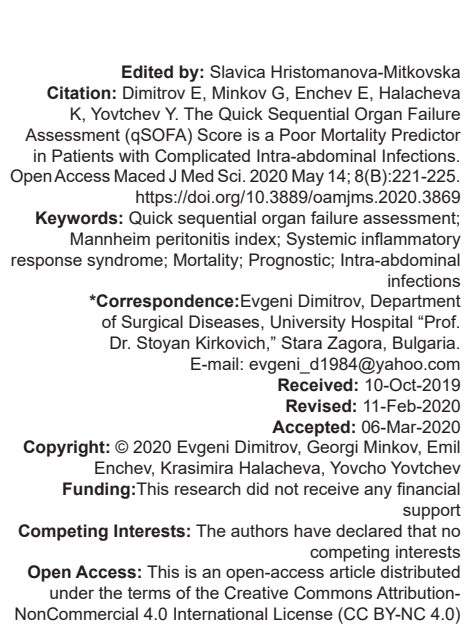

\begin{abstract}
BACKGROUND: Despite the evolution in surgical treatment and antimicrobial therapy in the last years the complicated intra-abdominal infections (cIAls) are still associated with high morbidity and mortality. Different scoring systems are already available for early prognostic evaluation and yet none has been widely accepted.

AIM: Our aim was to evaluate the prognostic accuracy of quick sequential organ failure assessment (qSOFA), one of the most recent scores, in patients with clAls.

MATERIALS AND METHODS: We studied retrospectively 110 patients with clAls admitted to the Department of Surgical Diseases (DSD) at University Hospital "Prof. Dr. Stoyan Kirkovich" Stara Zagora from January 2017 to July 2019. Area under receiver operating characteristics (AUROC) curves of systemic inflammatory response syndrome (SIRS), qSOFA, and Mannheim Peritonitis Index (MPI) were analyzed and a comparison of ROC curves was performed to explore their prognostic performance.

RESULTS: Twenty-five (22.7\%) patients died during hospitalization. qSOFA score showed poor prognostic accuracy (AUROC $=0.698,95 \% \mathrm{Cl}=0.566-0.829$ ), worse than MPI score (AUROC $=0.698$ vs. 0.844 ), but better than SIRS (AUROC $=0.698$ vs. 0.583 ). The qSOFA score $\geq 2$ points was observed with lack of sensitivity $(32.0 \%)$ as outcome predictor. ROC curve analysis showed prognostic inferiority of qSOFA compared to MPI (difference between areas $=0.146, p=0.0232$ )
\end{abstract}

CONCLUSION: In patients with clAls, quick-SOFA score was observed with poor prognostic performance.

\section{Introduction}

At the beginning of $21^{\text {st }}$ century complicated intra-abdominal infections (clAls) are still associated with unacceptably high mortality rates. In intensive care units (ICUs) they hold the second place after pneumonia as infectious cause of death [1]. Nowadays, more than $20 \%$ of sepsis in ICUs is a result of clAls [1].

clAls spread beyond the affected organ into the peritoneal cavity, which results in localized or diffuse peritonitis [2] and usually they are accompanied with sepsis. Sepsis is defined as a life-threatening organ dysfunction based on dysregulated host response to infection [3].

Early prognostic evaluation and appropriate treatment of patients with clAls are crucial for the final outcome. Various scoring systems have been applied over the years; so far however no score has shown enough prognostic accuracy in everyday practice. Most of the scoring systems are complex and difficult to calculate, require many clinical and laboratory measurements, and are used rarely outside of ICUs.
One of the most recent and least investigated prognostic scores is quick sequential organ failure assessment score (qSOFA). It is very easy to calculate based on only three clinical parameters at admission. qSOFA score was introduced by Sepsis-3 definitions task force [3] as prognostic tool that can promptly identify at the bedside patients with suspected infection who are likely to have a prolonged ICU stay or high risk for unfavorable outcome. qSOFA score was found superior to previous sepsis criteria for outcome prediction - in the emergency department (ED) [4], [5] and in patients with suspected infection outside ICU [6].

However, qSOFA showed lack of sensitivity as outcome predictor in several studies. Low sensitivity was observed in septic patients from Medical Admission Unit [7], in patients with acute infectious diseases [8], and in patients with infection presented to ED [9].

The insufficient data about the predictive value of qSOFA in patients with clAls and the increasing alert of its poor performance as mortality predictor in different clinical settings led us to the decision to evaluate the prognostic accuracy of qSOFA in surgical patients with clAls. 


\section{Materials and Methods}

\section{Design and participants}

This retrospective study was performed in the Department of Surgical Diseases at University Hospital "Prof. Dr. Stoyan Kirkovich" Stara Zagora. The medical records of 110 adult patients admitted to DSD from the ED who required emergency surgery for clAls from January 2017 to July 2019 were reviewed. None of the patients were suitable for percutaneous drainage or any other method of non-operative treatment. For this time interval, the admitted patients with diagnosis clAls were 131. Missing data about some clinical parameters were found in 18 patients, two patients died preoperatively, and one was under 18 years old. Finally, 110 patients were included in the study.

\section{Data collection}

We collected laboratory measurements, clinical information, clinical outcomes, and demographic data from hospital medical records of the studied patients.

\section{Scoring systems}

The quick-SOFA score includes three criteria - a systolic blood pressure (SBP) $\leq 100 \mathrm{~mm} \mathrm{Hg}$, a respiratory rate $(R R) \geq 22$ breaths $/ \mathrm{min}$, and a Glasgow Coma Scale (GCS) $<15$. A positive qSOFA score is $\geq 2$ out of 3 points [3]. Systemic inflammatory response syndrome (SIRS) includes four criteria - a heart rate $>90 / \mathrm{min}$, a tachypnea $>20 / \mathrm{min}$, a temperature $<36^{\circ} \mathrm{C}$ or $>38^{\circ} \mathrm{C}$, and leukocytes count $<4000 / \mathrm{mm}^{3}$ or $>12,000 / \mathrm{mm}^{3}$. Positive SIRS is defined as $\geq 2$ out of four signs [10]. SIRS and qSOFA were calculated based on patients' clinical data at admission. Mannheim Peritonitis Index (MPI) was calculated based on eight clinical parameters postoperatively [11] (Table 1).

Table 1: MPI (0-47 score)

\begin{tabular}{ll}
\hline Risk factor & Points \\
\hline Age $>50$ years & 5 \\
Female & 5 \\
Organ failure & 7 \\
Malignancy & 4 \\
Preoperatively duration of peritonitis $>24 \mathrm{~h}$ & 4 \\
Origin of sepsis non-colonic & 4 \\
Diffuse peritonitis & 6 \\
Exudate & \\
$\quad$ Clear & 0 \\
Purulent & 6 \\
Fecal & 12 \\
\hline
\end{tabular}

\section{Statistical analysis}

Sensitivity and specificity analysis and area under receiver operating characteristics (AUROC) for outcome prediction were evaluated for each score. De Long's method was used for comparison of the ROC curves. We evaluated the association between scoring systems and clinical outcome using bivariate correlation analysis and Spearman correlation coefficient. Continuous variables were expressed as mean (SD) or median (range) and categorical variables were expressed as frequency (\%). Comparisons were made by Mann-Whitney U-test or Student's t-test for continuous variables and by Chi-square test or Fisher exact test for categorical variables. $p<0.05$ was considered statistically significant. Statistical analysis was performed using SPSS Statistics 19.0 (IBM, Chicago, Illinois, USA) and MedCalc 14.8.1 (MedCalc Software, Ostend, Belgium).

\section{Results}

\section{Patients characteristics}

Of the total of 110 patients, $25(22.7 \%)$ died in hospital. Their average age was higher than survivors $(74.80 \pm 12.64$ vs. $56.84 \pm 18.89, p<0.0001)$. Significant differences between non-survivors and survivors were found according to the spread $(p=0.016)$ and site of infection $(p=0.041)$, exudate $(p=0.007)$, presence of chronic renal failure $(p=0.004)$, and malignancy $(p=0.002)$. We observed no significance according to gender $(p=0.693)$, presence of arterial hypertension $(p=0.353)$, and diabetes $(p=1.00)$ (Table 2$)$.

\section{Clinical parameters and scoring systems}

Eleven patients $(10.0 \%)$ had GCS $<15$ at admission, only one survived ( $p<0.0001)$. Tachypnea $\geq 22 / \mathrm{min}$ was found in 19 patients $(17.3 \%)$, nine died $(p=0.013)$. Twenty-one patients $(19.1 \%)$ had SBP $\leq 100 \mathrm{mmHg}$, nine died $(p=0.021)$. Thirty-four patients had heart rate $>90 / \mathrm{min}$, and 12 of them were nonsurvivors $(p=0.035)$. A positive SIRS showed no significant prognostic value $(p=0.172)$. The qSOFA score in survivors and non-survivors differs significantly $(p<0.0001)$. Eleven patients $(10.0 \%)$ had qSOFA $\geq 2$ and only three of them survived $(p<0.0001)$. Non-survivors had higher MPI score than survivors (30[26-35.5] vs. 21[16-25]). Eighty percent of non-survivors had MPI $>25$ points $(p<0.0001)$ (Table 3 ).

\section{Sensitivity, Specificity, and AUROCs}

Among the surveyed scores, we found SIRS as the worst mortality predictor (AUROC $=0.583,95 \%$ $\mathrm{Cl}=0.447-0.720)$, qSOFA showed better predictive performance (AUROC $=0.698,95 \% \mathrm{Cl}=0.566-0.829$ ), and MPI score was observed with the best prognostic value $($ AUROC $=0.844,95 \% \mathrm{Cl}=0.763-0.924)$ (Figure 1). The identified sensitivity and specificity for MPI threshold $>25$ points were $80.0 \%$ and $77.6 \%$, for qSOFA higher or equal to 2 points $-32.0 \%$ and $96.5 \%$, and for SIRS higher or equal to 2 points $-40.0 \%$ and $70.6 \%$, respectively (Table 4 ). 
Table 2: Patients characteristics

\begin{tabular}{|c|c|c|c|c|}
\hline Variable & Total population & Survivors $(\mathrm{n}=85)$ & Non-survivors $(n=25)$ & $p$-value \\
\hline Age, years $\pm S D$ & $60.92 \pm 19.17$ & $56.84 \pm 18.89$ & $74.80 \pm 12.64$ & $<0.0001$ \\
\hline Sex, n (\%) male/female & $61(55.5) / 49(45.5)$ & $48(78.7) / 37(75.5)$ & $13(21.3) / 12(24.5)$ & 0.693 \\
\hline \multicolumn{5}{|l|}{ Site, $\mathrm{n}(\%)$} \\
\hline Appendix & $27(24.5)$ & $25(29.4)$ & $2(8.0)$ & 0.041 \\
\hline Gallbladder & $26(23.6)$ & 20 (23.5) & $6(24.0)$ & \\
\hline Stomach/duodenum & $24(21.8)$ & $18(21.2)$ & $6(24.0)$ & \\
\hline Large bowel & $18(16.4)$ & $10(11.8)$ & $8(32.0)$ & \\
\hline Small bowel & $2(18.0)$ & $1(1.2)$ & $1(4.0)$ & \\
\hline Gynecological & $7(6.4)$ & $7(8.2)$ & $0(0)$ & \\
\hline Other & $6(5.5)$ & $4(4.7)$ & $2(8.0)$ & \\
\hline \multicolumn{5}{|l|}{ Spread, n (\%) } \\
\hline Local peritonitis & $40(36.4)$ & $36(42.4)$ & $4(16.0)$ & 0.016 \\
\hline Diffuse peritonitis & 70 (63.6) & 49 (57.6) & $21(84.0)$ & \\
\hline \multicolumn{5}{|l|}{ Exudate, $\mathrm{n}(\%)$} \\
\hline Clear & $21(19.1)$ & $19(22.4)$ & $2(8.0)$ & 0.007 \\
\hline Purulent & 84 (76.4) & 65 (76.5) & $19(76.0)$ & \\
\hline Fecal & $5(4.5)$ & $1(1.2)$ & $4(16.0)$ & \\
\hline \multicolumn{5}{|l|}{ Comorbidity, n (\%) } \\
\hline Diabetes & $13(11.8)$ & $10(11.8)$ & $3(12.0)$ & 1.000 \\
\hline Hypertension & $44(40.0)$ & $32(37.6)$ & $12(48.0)$ & 0.353 \\
\hline Malignancy & $16(14.5)$ & $7(8.2)$ & $9(36.0)$ & 0.002 \\
\hline Chronic renal failure & $9(8.2)$ & $3(3.5)$ & $6(24.0)$ & 0.004 \\
\hline
\end{tabular}

Table 3: Clinical parameters and scoring systems

\begin{tabular}{lllll}
\hline Variable & $\begin{array}{l}\text { Total } \\
\text { population }\end{array}$ & $\begin{array}{l}\text { Survivors } \\
(\mathrm{n}=85)\end{array}$ & $\begin{array}{l}\text { Non-survivors } \\
(\mathrm{n}=25)\end{array}$ & p-value \\
\hline SBP $\leq 100 \mathrm{mmHg}, \mathrm{n}(\%)$ & $21(19.1)$ & $12(14.1)$ & $9(36.0)$ & 0.021 \\
Heart rate $>90 / \mathrm{min}, \mathrm{n}(\%)$ & $34(30.9)$ & $22(25.9)$ & $12(48.0)$ & 0.035 \\
$\mathrm{RR} \geq 22 / \mathrm{min}, \mathrm{n}(\%)$ & $19(17.3)$ & $10(11.8)$ & $9(36.0)$ & 0.013 \\
GCS $<15, \mathrm{n}(\%)$ & $11(10.0)$ & $1(1.2)$ & $10(40.0)$ & $<0.0001$ \\
SIRS, $\mathrm{n}(\%)$ & $36(32.7)$ & $25(29.4)$ & $11(44.0)$ & 0.172 \\
qSOFA $\geq 2, \mathrm{n}(\%)$ & $11(10.0)$ & $3(3.5)$ & $8(32.0)$ & $<0.0001$ \\
qSOFA, $\mathrm{n}(\%)$ & & & & \\
0 & $77(70.0)$ & $66(77.6)$ & $11(44.0)$ & $<0.0001$ \\
1 & $22(20.0)$ & $16(18.8)$ & $6(24.0)$ & \\
2 & $6(5.5)$ & $3(3.5)$ & $3(12.0)$ & \\
3 & $5(4.5)$ & $0(0)$ & $5(20.0)$ & \\
MPI, points (IQR) & $21(18.8-30)$ & $21(16-25)$ & $30(26-35.5)$ & $<0.0001$ \\
MPI >25, n (\%) & $39(35.5)$ & $19(22.4)$ & $20(80.0)$ & $<0.0001$ \\
\hline
\end{tabular}

MPI: Mannheim Peritonitis Index, qSOFA: Quick sequential organ failure assessment, SIRS: Systemic inflammatory

Using pairwise comparison analysis of ROC curves, we found significant differences between scores. The MPI showed prognostic superiority than SIRS (difference between areas $=0.260,95 \%$ $\mathrm{Cl}=0.109-0.412, \mathrm{p}=0.0008$ ) and qSOFA (difference between areas $=0.146,95 \% \mathrm{Cl}=0.0199-0.272$, $p=0.0232$ ). qSOFA was better outcome predictor than SIRS (difference between areas $=0.114,95 \%$ $\mathrm{Cl}=0.0249-0.226, \mathrm{p}=0.0451$ ) (Table 5).

Table 4: Sensitivity, specificity, and AUROCs

\begin{tabular}{llll}
\hline Variable & Sensitivity, $\%$ & Specificity, $\%$ & AUROC \\
\hline SIRS $\geq 2$ & 40.0 & 70.6 & $0.583(0.447-0.720)$ \\
qSOFA $\geq 2$ & 32.0 & 96.5 & $0.698(0.566-0.829)$ \\
MPI $>25$ & 80.0 & 77.6 & $0.844(0.763-0.924)$ \\
\hline
\end{tabular}

AUROC: Area under receiver operating characteristic, MPI: Mannheim Peritonitis Index, qSOFA: Quick sequential organ failure assessment, SIRS: Systemic inflammatory response syndrome.

\section{Correlations}

We found weak correlation between qSOFAand outcome $(r=0.356, p<0.0001)$, moderate correlation between MPI and outcome ( $r=0.500, p<0.0001)$, and very weak correlation with no significance between SIRS and outcome $(r=0.128, p=0.181)$ (Table 6).

\section{Discussion}

The enormous diversity of patient groups affected by clAls makes the recommendation of a

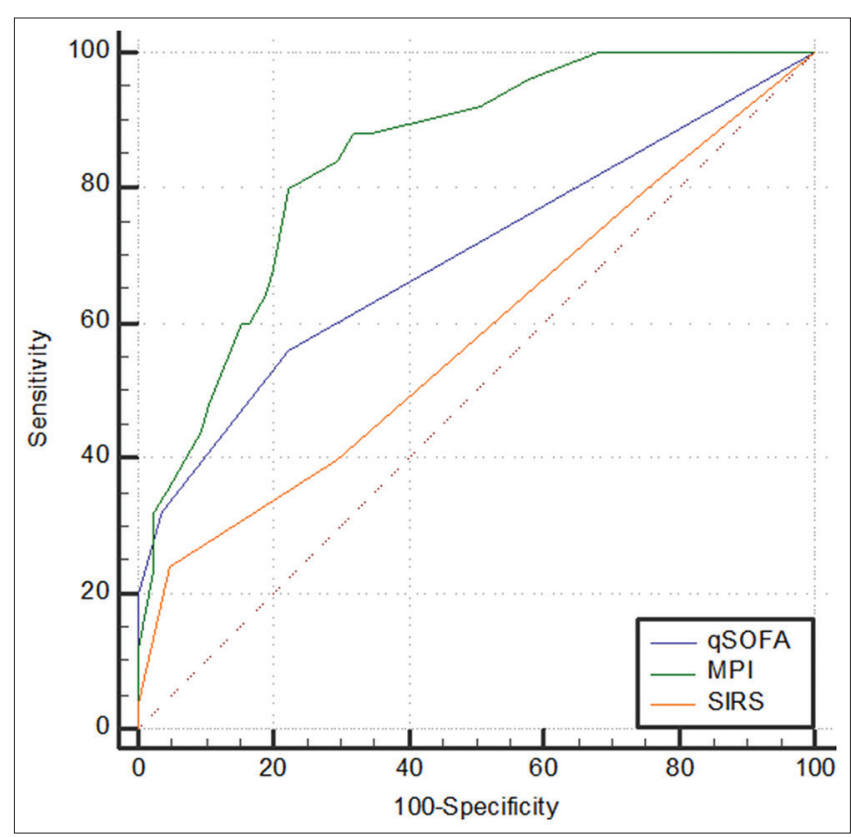

Figure 1: Comparison of ROC curves

general treatment algorithm not an easy task. By predicting the outcome of each patient with clAl, there has to be considered a wide range of unfavorable factors such as poor nutrition, immunosuppression, nosocomial pathogens, pre-existing diseases, advanced age, diffuse peritonitis, delayed treatment, septic shock, organ failures, and poor source control [12].

Table 5: Pairwise comparison of ROC curves

\begin{tabular}{llll}
\hline Comparison criteria & MPI qSOFA & qSOFA $~$ SIRS & MPI SIRS \\
\hline Difference between areas $^{\text {Standard Error }}{ }^{\mathrm{C}}$ & 0.146 & 0.114 & 0.260 \\
$95 \% \mathrm{Cl}$ & 0.0643 & 0.0571 & 0.0772 \\
Significance & $0.0199-0.272$ & $0.0249-0.226$ & $0.109-0.412$ \\
\hline MPI: Mannheim Peritonitis Index, qSOFA: Quick sequential organ failure assessment, SIRS: Systemic \\
inflammatory response syndrome.
\end{tabular}

Despite the identical conservative and surgical management some patients' populations distinguish with a high mortality rates. Nevertheless, early prognosis and timely therapy in these patients enhance the chances of favorable outcome [13]. All these facts indicate the necessity of significant methods that could contribute for early prognostic assessment and determine the aggressiveness of treatment regimens. A 
large number of researchers are still trying to deal with these problems focusing on the predictive reliability of different prognostic scoring systems.

\section{Table 6: Correlations}

\begin{tabular}{llll}
\hline Outcome & qSOFA & SIRS & MPI \\
\hline Correlation coefficient & $\mathrm{r}=0.356$ & $\mathrm{r}=0.128$ & $\mathrm{r}=0.500$ \\
Significance & $\mathrm{p}<0.0001$ & $\mathrm{p}=0.181$ & $\mathrm{p}<0.0001$ \\
\hline MPI: Mannheim Peritonitis Index, qSOFA: Quick sequential organ failure assessment, SIRS: Systemic
\end{tabular}

inflammatory response syndrome.

One of these scoring systems, the full SOFA (fSOFA) score showed reliable characteristics over the years and nowadays it is a part of the new SEPSIS 3 DEFINITIONS [3]. This score showed better prognostic accuracy than SIRS or qSOFA in adult patients with suspected infection admitted to ICU [14].

However, fSOFA is not a simple score - it needs numerous clinical and laboratory measurements for calculation; moreover, outside ICUs in everyday practice this score is hardly used. As simplified version of fSOFA, Sepsis-3 Group introduced qSOFA score for easier identification of ED patients with infection and higher risk of death.

However, in clAls qSOFA showed low sensitivity as prognostic score. Only two authors (to the best of our knowledge) explored prognostic value of qSOFA score in surgical patients exclusively. Jung et al. [15] investigated qSOFA score in 457 surgical patients with clAls. Authors observed sensitivity of $46 \%$ for qSOFA $\geq 2$ as outcome predictor. The second study with surgical patients of Raimondo et al. [16] reported even worse sensitivity - only $14.3 \%$. We also observed low sensitivity of qSOFA as mortality predictor $-32 \%$.

Jiang et al. [17] in their meta-analysis identified lack of sensitivity (42\%) of this score in ED patients with infection.

Various studies with non-surgical patients presented to ED showed higher sensitivity of qSOFA. The highest sensitivity of qSOFA $\geq 2-90 \%$ was found in the study of Finkelsztein et al. [6] with noncritically ill patients. A sensitivity of $70 \%$ for qSOFA $\geq 2$ was reported by Freund et al. [4] in ED patients with suspected infection. Osatnik et al. [5] observed in their study sensitivity of $63.6 \%$.

Although its limitations, qSOFA score has one serious advantage - a perfect ability to recognize the patients who will survive - the observed specificity for qSOFA $\geq 2$ in our study was $96.5 \%$; the other two studies with surgical patients with clAls showed also very high specificity - 86\% Jung et al. [15] and 98.3\% Raimondo et al. [16]. A meta-analysis [17] from 2018 with infected patients from ED reported also very high specificity of $88 \%$ for outcome prediction.

We made an interesting observation in the present study - a change in patient mental status is strongly associated with fatal outcome. Of the total of eleven patients with GCS $<15$, ten died ( $p<0.0001)$ and they represented $40 \%$ of non-survivors. Perhaps the altered mental status may represent an important factor for early prognosis in patients with clAls. Unlike our study, Jung et al. [15] observed that only 4 out of 15 patients with GCS < 15 were non-survivors. The observations of Freund et al. [4] were similar to our results $-56 \%$ of ED patients with altered mental status died.

In our study, qSOFA showed poor prognostic value $(A U R O C=0.698)$. The other two studies with surgical patients reported also not good prognostic accuracy of qSOFA - Jung et al. [15] (AUROC = 0.717) and Raimondo et al. [16] (AUROC = 0.722). Osatnik et al. [5] in patients admitted to the ED with suspected infection observed similar prognostic performance of qSOFA (AUROC = 0.71).

Good prognostic performance was established by Freund et al. [4] in other study with ED patients AUROC $=0.80$.

All of the performed statistical analyses in the present study showed prognostic inferiority of qSOFA compared to MPI, one of the oldest scores for mortality prediction in patients with acute peritonitis. ROC curve analysis revealed better prognostic value of $\mathrm{MPI}$ (AUROC $=0.844)$ compared to qSOFA (AUROC = 0.698). The pairwise comparison of ROC curves of MPI and qSOFA scores revealed prognostic superiority of MPI (difference between areas $=0.146, p=0.0232$ ). The bivariate correlation analysis pointed weak correlation between qSOFA and outcome $(r=0.356)$ and stronger correlation between MPI and outcome $(r=0.500)$. No other study (to the best of our knowledge) investigated correlations between qSOFA, MPI and outcome and compared prognostic values of both scores in patients with clAl.

\section{Limitations}

The present study has several limitations. First of all, it was a retrospective study. The investigated sample size was small. Other important limitation is that this was a single-center trial.

\section{Conclusion}

In patients with clAls qSOFA score showed poor prognostic performance. Although its simplicity, easy calculation and high specificity this score could not recognize nearly two-third of the patients with higher risk of death. Maybe it is time to announce the uselessness of qSOFA as prognostic score in patients with clAls and to pay attention to other scoring systems. 


\section{References}

1. Vincent J, Rello J, Marshall J, Silva E, Anzueto A, Martin CD, et al. International study of the prevalence and outcomes of infection in intensive care units. JAMA. 2009;302(21):2323-9. https://doi.org/10.1001/jama.2009.1754

PMid:19952319

2. Lopez N, Kobayashi L, Coimbra R. A comprehensive review of abdominal infections. World J Emerg Surg. 2011;6(1):7.

3. Singer M, Deutschman CS, Seymour CW, Shankar-Hari M, Annane $\mathrm{D}$, Bauer $\mathrm{M}$, et al. The third international consensus definitions for sepsis and septic shock (sepsis-3). JAMA. 2016;315(8):801-10. https://doi.org/10.1001/jama.2016.0288 PMid:26903338

4. Freund $\mathrm{Y}$, Lemachatti $\mathrm{N}$, Krastinova $\mathrm{E}$, van Laer $\mathrm{M}$, Claessens YE, Avondo A, et al. Prognostic accuracy of sepsis-3 criteria for in-hospital mortality among patients with suspected infection presenting to the emergency department. JAMA. 2017;317(3):301-8. https://doi.org/10.1001/jama.2016.20329 PMid:28114554

5. Osatnik J, Tort-Oribea B, Folco J, Sosa A, Ivulich D, Kleinert MM, et al. Predictive performance of quick sequential organ failure assessment scoring in an Argentinian hospital. J Clin Diagnostic Res. 2018;12(10):OC22-6. https://doi.org/10.7860/ jcdr/2018/37018.12150

6. Finkelsztein EJ, Jones DS, Ma KC, Pabón MA, Delgado T, Nakahira K, et al. Comparison of qSOFA and SIRS for predicting adverse outcomes of patients with suspicion of sepsis outside the intensive care unit. Crit Care. 2017;21:73. https://doi. org/10.1186/s13054-017-1658-5

PMid:28342442

7. Goulden R, Hoyle MC, Monis J, Railton D, Riley V, Martin P, et al. qSOFA, SIRS and news for predicting in hospital mortality and ICU admission in emergency admissions treated as sepsis. Emerg Med J. 2018;35(6):345-9. https://doi.org/10.1136/ emermed-2017-207120

PMid:29467173

8. Gaini S, Relster MM, Pedersen C, Johansen IS. Prediction of 28-days mortality with sequential organ failure assessment (SOFA), quick SOFA (qSOFA) and systemic inflammatory response syndrome (SIRS)-a retrospective study of medical patients with acute infectious disease. Int J Infect Dis. 2019;78:1-7. https://doi.org/10.1016/j.ijid.2018.09.020 PMid:30267939

9. Askim A, Moser F, Gustad LT, Stene H, Gundersen M, Åsvold BO et al. Poor performance of quick-SOFA (qSOFA) score in predicting severe sepsis and mortality-a prospective study of patients admitted with infection to the emergency department. Scand J Trauma Resusc Emerg Med. 2017;25(56):2-9. https://

\section{doi.org/10.1186/s13049-017-0399-4}

10. Bone RC, Balk RA, Cerra FB, Dellinger RP, Fein AM, Knaus WA et al. Definitions for sepsis and organ failure and guidelines for the use of innovative therapies in sepsis. The ACCP/ SCCM consensus conference committee. American college of chest physicians/society of critical care medicine. Chest. 1992;101(6):1644-55. https://doi.org/10.1378/chest.101.6.1644 PMid:1303622

11. Wacha H, Linder MM, Feldman U, WeschG, Gundlach E, Steifensand RA. Mannheim peritonitis index-prediction of risk of death from peritonitis: Construction of a statistical and validation of an empirically based index. Theor Surg. 1987;1:169-77. https://doi.org/10.1007/978-3-642-73008-5_6

12. Sartelli M, Abu-Zidan FM, Catena F, Griffiths EA, Di Saverio S, Coimbra R, et al. Global validation of the WSES sepsis severity score for patients with complicated intra-abdominal infections: A prospective multicentre study (WISS study). World J Emerg Surg. 2015;10:61. https://doi.org/10.11622/smedj.2018120 PMid:26677396

13. Otero RM, Nguyen HB, Huang DT, Gaieski DF, Goyal M, Gunnerson KJ, et al. Early goal-directed therapy in severe sepsis and septic shock revisited: Concepts, controversies, and contemporary findings. Chest. 2006;130(5):1579-95. https://doi. org/10.1378/chest.130.5.1579

PMid: 17099041

14. Raith EP, Udy AA, Bailey M, McGloughlin S, Maclsaac C, Bellomo R, et al. Prognostic accuracy of the SOFA score, SIRS criteria, and qSOFA score for in-hospital mortality among adults with suspected infection admitted to the intensive care unit. JAMA. 2017;317(3):290-300. https://doi.org/10.1001/ jama.2016.20328

PMid:28114553

15. Jung YT, Jeon J, Park JY, Kim MJ, Lee SH, Lee JG. Addition of lactic acid levels improves the accuracy of quick sequential organ failure assessment in predicting mortality in surgical patients with complicated intra-abdominal infections: A retrospective study. World J Emerg Surg. 2018;13:14. https:// doi.org/10.1186/s13017-018-0173-6 PMid:29563963

16. Raimondo $S$, Sartelli $M$, Coccolini F, Fugazzola $P$, Bova $R$, Tomasoni $\mathrm{M}$, et al. Which prognostic score for abdominal sepsis? Analysis of final results of PIPAS (physiological indicators for prognosis in abdominal sepsis) study in a single center. J Peritoneum. 2018;3:106. https://doi.org/10.4081/ joper.2018.106

17. Jiang J, Yang J, Mei J, Jin Y, Lu Y. Head-to-head comparison of qSOFA and SIRS criteria in predicting the mortality of infected patients in the emergency department: A meta-analysis. Scand J Trauma Resusc Emerg Med. 2018;26(1):56. https://doi. org/10.1186/s13049-018-0527-9

PMid:29996880 